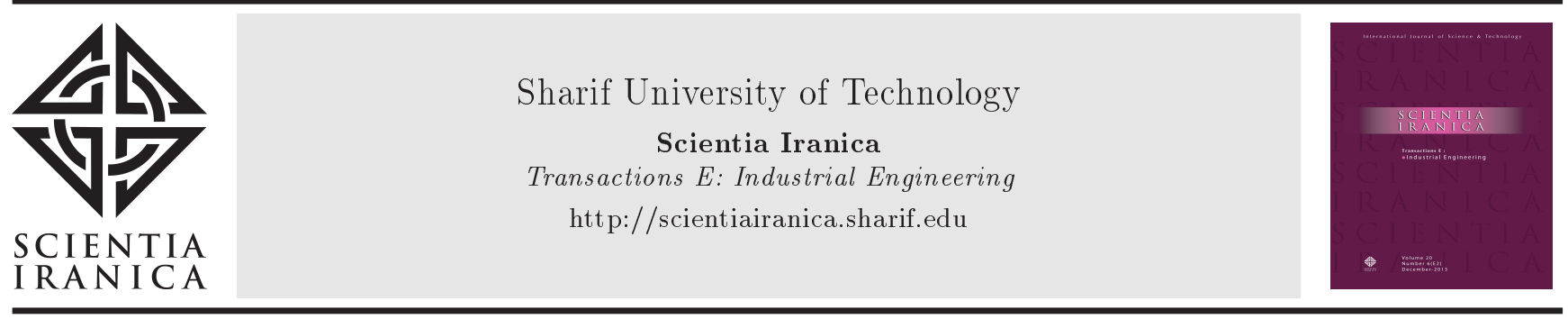

\title{
The NDEA-MOP model in the presence of negative data using fuzzy method
}

\author{
K. Kianfar ${ }^{\mathrm{a}}$, M. Ahadzadeh Namin ${ }^{\mathrm{b}, *}$, A. Alam Tabriz ${ }^{\mathrm{c}}$, E. Najafi ${ }^{\mathrm{a}}$, and \\ F. Hosseinzadeh Lotfi ${ }^{\mathrm{d}}$ \\ a. Department of Industrial Engineering, Science and Research Branch, Islamic Azad University, Tehran, Iran. \\ b. Department of Mathematics, Shahr-e-Qods Branch, Islamic Azad University, Tehran, Iran. \\ c. Department of Management, Shahid Beheshti University, Tehran, Iran. \\ d. Department of Mathematics, Science and Research Branch, Islamic Azad University, Tehran, Iran.
}

Received 13 February 2016; received in revised form 30 October 2016; accepted 15 November 2016

\section{KEYWORDS}

Data envelopment analysis;

Network DEA;

Semi-positive data;

Semi-negative data;

Overall efficiency;

Fuzzy method.

\begin{abstract}
In this study, the Multi-Objective Programming (MOP) method was used to solve Network DEA (NDEA) models with negative data, which consisted of semi-negative and semi-positive input and output. At first, two stage and, then, $k$-stage production models were formulated with consideration of negative data. In the multi-objective programming, two separate objective functions, including the divisional efficiencies and the overall efficiency of the organization, were modeled. In comparison to conventional DEA with negative data, the advantage of the proposed NDEA models is consideration of intermediate processes and products, in order to calculate overall efficiency of the organization. However, in conventional DEA, sub-stages of the organizations are neglected. To measure the efficiencies of an organization regarding interactive internal process, two case studies were investigated by application of the NDEA-MOP method with negative data. Case study 1 was focused on units with two stages having semi-negative and semipositive indices. In case study 2, units with three stages were evaluated. These units had semi-negative and semi-positive indices, too. The overall efficiency of each unit was calculated using the proposed models. Fuzzy approach as a solution procedure was applied.

(C) 2018 Sharif University of Technology. All rights reserved.
\end{abstract}

\section{Introduction}

Nowadays, performance assessment of industrial and economical units plays an important role in achieving managerial success and continuous progress. Data Envelopment Analysis (DEA) is a nonparametric method used to analyze and evaluate the performance of De-

\footnotetext{
*. Corresponding author.

E-mail addresses: kianoush.kianfar@gmail.com (K.

Kianfar); mahnazahadzadehnamin@gmail.com ( $M$.

Ahadzadeh Namin); a-tabriz@sbu.ac.ir (A. Alam Tabriz);

najafi1515@yahoo.com (E. Najafi); Farhad@hosseinzadeh.ir

(F. Hosseinzadeh Lotfi)
}

doi: $10.24200 /$ sci. 2017.4413 cision Making Units (DMUs), which converts multiple inputs into multiple outputs and takes the qualitative and quantitative measures into account. In recent years, extensive application of DEA has been observed in several contexts such as health care, education, manufacturing, retailing, banking, etc. In the conventional DEA model, two types of models, namely, the aggregation and separation approaches, are applied to measure efficiencies. In the aggregation model, divisions are aggregated into a single company, the DMU is evaluated as a black box, and the internal linking activities are neglected. Therefore, it is not possible to evaluate the performance of an individual division. In the separation model, each division in a DMU is considered as a separate unit and the 
linking activities between divisions are completely ignored. Thus, efficiencies of the organization's linking processes via both the mentioned methods cannot be evaluated [1]. The Network DEA (NDEA) model was proposed by Lewis and Sexton [2] to overcome the weakness of the traditional DEA model. This model had a multi-stage structure, which accounted for both divisional efficiencies and the overall efficiency in a unified framework. Also, it considered internal interaction within DMUs, where the intermediate measures among the stages play crucial roles in evaluation of the efficiency. In recent years, the attention of a large number of researchers has been drawn to efficiency assessment in multi-stage production processes, where each DMU transforms some external inputs to final outputs by the intermediate products. Details of some researches in this field can be found in [3-6]. The first DEA model, namely, CCR, was proposed by Charnes et al. [7] with assumption of constant-returns-to-scale. The evolutionary form of this model, named BCC [8], was proposed by extending to variable-returns-toscales. In BCC, $n$ DMUs are considered $(j=1, \ldots, n)$ for assessment. Each DMU consumes $m$ inputs $(i=$ $1, \ldots, m)$ and produces $s$ outputs $(r=1, \ldots, s)$, denoted by $\left(x_{i j}, x_{2 j}, \ldots, x_{m j}\right)$ and $\left(y_{1 j}, y_{2 j}, \ldots, y_{s j}\right)$, respectively. The efficiency of DMUk can be calculated by the CCR and BCC models as Eqs. (1) and (2):

\section{CCR:}

$$
\begin{aligned}
& \operatorname{Max} \quad E_{k}=\frac{\sum_{r=1}^{s} u_{r} y_{r k}}{\sum_{i=1}^{m} v_{i} x_{i k}}, \\
& \text { s.t. } \quad \frac{\sum_{r=1}^{s} u_{r}}{\sum_{i=1}^{m} v_{i} x_{i j}} \leq 1, \quad j=1,2, \ldots, n, \\
& u_{r}, v_{i} \geq \varepsilon, \quad r=1,2, \ldots, s ; \quad i=1,2, \ldots, m .
\end{aligned}
$$

BCC:

$$
\begin{aligned}
& \operatorname{Max} \quad E_{k}=\frac{\sum_{r=1}^{s} u_{r} y_{r k}-u_{0}}{\sum_{i=1}^{m} v_{i} x_{i k}}, \\
& \text { s.t. } \quad \frac{\sum_{r=1}^{s} u_{r} y_{r j}-u_{0}}{\sum_{i=1}^{m} v_{i} x_{i j}} \leq 1, \quad j=1,2, \ldots, n \\
& u_{r}, v_{i} \geq \varepsilon, \quad r=1,2, \ldots, s ; \quad i=1,2, \ldots, m,
\end{aligned}
$$

$u_{0} \quad$ Unrestricted in sign.
In Eqs. (1) and (2), $E_{k}$ is the objective function, which is maximized for every $\mathrm{DMU}_{\mathrm{k}}$, individually; $u_{r}$ and $v_{i}$ are weights of the outputs and inputs, respectively; $x_{i k}$ and $y_{r k}$ are the $i$-th input and $r$-th output of $\mathrm{DMU}_{k} ; \varepsilon$ is a small positive value, which indicates positive weights; and $\mathrm{u}_{0}$ is the intercept of the production function in the $\mathrm{BCC}$ model. Previous researches have documented different methods for solving network DEA. Cheng et al. [9] derived a common set of weights by Multi-Objective Programming (MOP) model based on a compensatory Data Envelopment Analysis (DEA) model, in order to rank all DMUs. In order to solve it, the MOP model was transformed into a Single-Objective Programming (SOP) using a fuzzy programming method. Thereafter, the SOP model was solved by the proposed approximation algorithm. Kao et al. [10] proposed the Multi-Objective Programming (MOP) method in order to solve Network DEA (NDEA). Two types of NDEA-MOP models, namely, BCC-MOP and CCR-MOP, were assessed. Divisional and the overall efficiencies of the organization were measured without neglecting the efficiencies of its subunits. Matin and Azizi [11] measured performance of production systems by a new unified generalized Network DEA model when interrelationships between individual sub-processes were considered. General Network DEA model was evaluated by some illustrative numerical examples. Wang et al. [12] constructed two-stage DEA model and then used a fuzzy multiobjective for evaluating the performance of US Bank Holding Companies (BHCs). This paper analyzed the relationship between BHCs performance and their Intellectual Capital (IC). Despotis and Koronakos [13] assessed efficiency of a two-stage network using a novel DEA approach. In the proposed method, unique and unbiased efficiency scores for the individual stages were calculated. Afterwards, a posteriori aggregation method was applied in order to compose stages to obtain the efficiency of the overall system. Halkos et al. [14] reviewed the classification of two-stage DEA models, as well as their mathematical formulations, and main applications. Simple cases, such as the two-stage models, and general models, such as the network DEA models, were analyzed. These models were categorized into four models, namely, independent, connected, relational, and game theoretic two-stage DEA. Lee and Li [15] studied fuzzy multiple-objective programming and compromise programming with Pareto optimum. In recent times, evolutionary algorithms have become a widely used methodology in MOP. The main aim of this study was to solve network DEA by applying the Multi-Objective Programming (MOP) method. At first, a two-stage production system was assumed in order to convert some input products in the first stage and use these outputs as inputs to the second stage for producing final outputs. In this paper, at first, negative 
data is considered for the proposed NDEA model, which consists of semi-negative and semi-positive input and output. Thereafter, the $k$-stage production process with consideration of negative data is formulated by MOP. MOP is concerned with finding the solutions in which a set of objective functions are simultaneously optimized, meaning that it is not possible to improve any objective without degrading some others. Many practical applications such as pattern classification can be posed as MOP problems.

According to the CCR and BCC models, objective functions including the overall and divisional efficiencies within a DMU are optimized. Two case studies are evaluated to indicate the benefits of NDEAMOP. In order to calculate efficiency of units in the presence of semi-negative and semi-positive indices, various models are presented. Also, different views are suggested in order to calculate the performance of two- and multi-stage units. The difference between this article and other articles is that the present study deals with two- and multi-stage units having semi-negative and semi-positive indices. In other words, this paper focuses on units which are combinations of these two states. The remainder of this paper is structured as follows: in Section 2, the method of Izadikhah and Farzipoor Saen [16] is discussed. In Section 3, twostage DEA model is discussed. In Section 4, a model for calculation of overall efficiency of units with two stages in the presence of semi-positive and semi-negative data is proposed. In Section 5, units are extended to $k$ stages and a method is stated for calculating the overall efficiency of units. Fuzzy approach [17] as a solution procedure is proposed in two sections. In Section 6, two case studies are implemented to examine the network DEA models with multi-objective programming. In Section 7 , results and discussion are given and the conclusion section is provided at the end of the paper.

\section{Non-radial efficiency of two-stage network DEA with negative data}

Consider $n$ units under assessment of $\mathrm{DMU}_{j}(j=$ $1, \ldots, n)$ with two-stage network structure as shown in Figure 1. Stage 1 consumes $X_{j}$ as input and produces $Z_{j}$ as output, and Stage 2 consumes $Z_{j}$ as input and produces $Y_{j}$ as output. Izadikhah and Farzipoor Saen [16], using the idea of Chen and Zhu [18], presented non-radial model to calculate efficiency of each stage and the overall efficiency of a unit in the presence

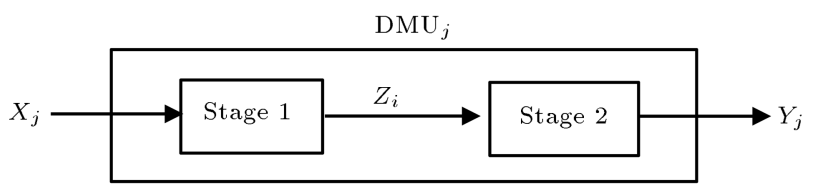

Figure 1. Two-stage network model. of negative data. This model for the evaluation of $\mathrm{DMU}_{p}$ unit is as follows:

Stage 1:

$$
\begin{aligned}
\operatorname{Max} & w_{1}\left(\frac{1}{m} \sum_{i=1}^{m} \frac{\theta_{i}}{\bar{\theta}_{p}}+\frac{1}{D} \sum_{d=1}^{D} \frac{\varphi_{d}}{\bar{\varphi}_{p}}\right) \\
+ & w_{2}\left(\frac{1}{D} \sum_{d=1}^{D} \frac{\alpha_{d}}{\bar{\alpha}_{p}}+\frac{1}{s} \sum_{r=1}^{s} \frac{B_{r}}{\bar{B}_{p}}\right),
\end{aligned}
$$

s.t.

$$
\begin{aligned}
& \sum_{j=1}^{n} \lambda_{j} x_{i j} \leq x_{i p}-\theta_{i}\left|x_{i p}\right| ; \quad i=1, \ldots, m, \\
& \sum_{j=1}^{n} \lambda_{j} z_{d j} \leq \tilde{z}_{d p}-\varphi_{d}\left|\tilde{z}_{d p}\right| ; \quad d=1, \ldots, D, \\
& \sum_{j=1}^{n} \lambda_{j}=1, \quad \lambda_{j} \geq 0 ; \quad j=1, \ldots, n .
\end{aligned}
$$

Stage 2:

$$
\begin{aligned}
& \sum_{j=1}^{n} \mu_{j} d_{d j} \leq \tilde{z}_{d p}-\alpha_{d}\left|\tilde{z}_{d p}\right| ; \quad d=1, \ldots, D, \\
& \sum_{j=1}^{n} \mu_{j} y_{r j} \geq y_{r p}+B_{r}\left|y_{r p}\right| ; \quad r=1, \ldots, s, \\
& \sum_{j=1}^{n} \mu_{j}=1, \quad \mu_{j} \geq 0 ; \quad j=1, \ldots, n,
\end{aligned}
$$

where:

$$
\begin{aligned}
& \bar{\theta}_{p}=\operatorname{Max}_{i}\left\{\frac{x_{i p}-x_{i L}}{\left|x_{i p}\right|} ; \quad x_{i p} \neq 0, \quad i=1, \ldots, m\right\}, \\
& \bar{\alpha}_{p}=\operatorname{Max}_{d}\left\{\frac{z_{d p}-z_{d L^{\prime}}}{\left|z_{d p}\right|} ; \quad z_{d p} \neq 0, \quad d=1, \ldots, D\right\}, \\
& \bar{\varphi}_{p}=\operatorname{Max}_{d}\left\{\frac{z_{d L}-z_{d p}}{\left|z_{d p}\right|} ; \quad z_{d p} \neq 0, \quad d=1, \ldots, D\right\}, \\
& \bar{B}_{p}=\operatorname{Max}_{r}\left\{\frac{y_{r L^{\prime}}-y_{r p}}{\left|y_{r p}\right|} ; \quad y_{r p} \neq 0, \quad r=1, \ldots, s\right\},
\end{aligned}
$$

and:

$$
\begin{aligned}
& x_{i L}=\operatorname{Min}_{j}\left\{x_{i j}\right\} ; \quad i=1, \ldots, m, \\
& z_{d L^{\prime}}=\operatorname{Min}_{j}\left\{z_{d j}\right\} ; \quad d=1, \ldots, D,
\end{aligned}
$$




$$
\begin{aligned}
& z_{d L}=\operatorname{Max}_{j}\left\{z_{d j}\right\} ; \quad d=1, \ldots, D, \\
& y_{r L^{\prime}}=\operatorname{Max}_{j}\left\{y_{r j}\right\} ; \quad r=1, \ldots, s .
\end{aligned}
$$

In the presented model, $w_{1}+w_{2}=1$ and overall efficiency of $\mathrm{DMU}_{p}$ are calculated as follows:

$$
\begin{gathered}
\theta^{*}=1-\left(\frac{w_{1}}{2}\left[\frac{1}{m} \sum_{i=1}^{m} \frac{\theta_{i}^{*}}{\bar{\theta}_{p}}+\frac{1}{D} \sum_{d=1}^{D} \frac{\varphi_{d}^{*}}{\bar{\varphi}_{p}}\right]\right. \\
\left.+\frac{w_{2}}{2}\left[\frac{1}{D} \sum_{d=1}^{D} \frac{\alpha_{d}^{*}}{\bar{\alpha}_{p}}+\frac{1}{s} \sum_{r=1}^{s} \frac{B_{r}^{*}}{\bar{B}_{P}}\right]\right), \\
\theta^{*}=1-\left[w_{1}\left(1-\theta_{1}^{*}\right)+w_{2}\left(1-\theta_{2}^{*}\right)\right],
\end{gathered}
$$

where $\theta_{1}^{*}$ and $\theta_{2}^{*}$ are efficiencies of Stages 1 and 2 , respectively.

Note that, in Model (3), $\tilde{z}$ is an unknown decision variable, which is calculated by solving the model. In this paper, a method is presented, which considers general state of a two-stage model (i.e., Stage 2 has external input) and $k$-stage model for non-negative indices.

\section{Two-stage DEA model}

Consider $n$ DMUs under assessment. Figure 1 shows the network structure of each DMU, where $X_{j}$ expresses input to the first stage and $Z_{j}$ represents output of the first stage, which is considered as input to the second stage, and $Y_{j}$ is taken into account as output of the second stage, where $X_{j}=\left(x_{1 j}, x_{2 j}, \ldots, x_{m j}\right)$, $Y_{j}=\left(y_{1 j}, y_{2 j}, \ldots, y_{s j}\right)$, and $Z_{j}=\left(z_{1 j}, z_{2 j}, \ldots, z_{t j}\right)$ are positive.

\section{Two-stage NDEA-CCR model in the presence of semi-positive and semi-negative data}

It is assumed that $I$ is input series, which is positive in all DMUs and $\bar{I}$ is input series, which is positive in some DMUs and negative in other DMUs, so that $m=|I|+|\bar{I}|$ and $I \cap \bar{I}=\phi$. Furthermore, $T$ is $z_{t j}$ series, which is positive in all decision making units and $\bar{T}$ is $z_{t j}$ series, which is positive in some units and negative in others as $t=|T|+|\bar{T}|$ and $T \cap \bar{T}=\phi$. In addition, $R$ is output series, which is positive in all DMUs, and $\bar{R}$ is output series, which is positive in some DMUs and negative in others such that $s=|R|+|\bar{R}|$ and $R \cap \bar{R}=\phi$. The efficiency of each DMU can be calculated as follows:

$$
E_{j}^{(1)}=\frac{\sum_{t \in T} \mu_{t} z_{t j}+\sum_{t \in \bar{T}} \bar{\mu}_{t} z_{t j}^{(1)}-\sum_{t \in \bar{T}} \hat{\mu}_{t} z_{t j}^{(2)}}{\sum_{i \in I} v_{i} x_{i j}+\sum_{i \in \bar{I}} \bar{v}_{i} x_{i j}^{(1)}-\sum_{i \in \bar{I}} \hat{v}_{i} x_{i j}^{(2)}},
$$

$$
E_{j}^{(2)}=\frac{\sum_{r \in R} u_{r} y_{r j}+\sum_{r \in \bar{R}} \bar{u}_{r} y_{r j}^{(1)}-\sum_{r \in \bar{R}} \hat{u}_{r} y_{r j}^{(2)}}{\sum_{t \in T} \mu_{t} z_{t j}+\sum_{t \in \bar{T}} \bar{\mu}_{t} z_{t j}^{(1)}-\sum_{t \in \bar{T}} \hat{\mu}_{t} z_{t j}^{(2)}},
$$

where $\mu_{t}, \bar{\mu}_{t}, \hat{\mu}_{t}, v_{i}, \bar{v}_{i}, \hat{v}_{i}, u_{r}, \bar{u}_{r}$, and $\hat{u}_{r}$ are weights of inputs and outputs and are all non-negative. In addition, for every $t \in \bar{T}$, we have $z_{t j}=z_{t j}^{(1)}-z_{t j}^{(2)}$ where $z_{t j}^{(1)} \geq 0, z_{t j}^{(2)} \geq 0$. Furthermore:

$$
z_{t j}^{(1)}=\left\{\begin{array}{ll}
z_{t j} & z_{t j} \geq 0 \\
0 & z_{t j}<0
\end{array} ; \quad z_{t j}^{(2)}= \begin{cases}0 & z_{t j} \geq 0 \\
-z_{t j} & z_{t j}<0\end{cases}\right.
$$

For every $r \in \bar{R}$, we have $y_{r j}=y_{r j}^{(1)}-y_{r j}^{(2)}$ where $y_{r j}^{(1)} \geq$ $0, y_{r j}^{(2)} \geq 0$ and:

$$
y_{r j}^{(1)}=\left\{\begin{array}{ll}
y_{r j} & y_{r j} \geq 0 \\
0 & y_{r j}<0
\end{array} ; \quad y_{r j}^{(2)}= \begin{cases}0 & y_{r j} \geq 0 \\
-y_{r j} & y_{r j}<0\end{cases}\right.
$$

In addition, for $i \in \bar{I}$, we have $x_{i j}=x_{i j}^{(1)}-x_{i j}^{(2)}$ where $x_{i j}^{(1)} \geq 0, x_{i j}^{(2)} \geq 0$ :

$$
x_{r j}^{(1)}=\left\{\begin{array}{ll}
y_{r j} & x_{i j} \geq 0 \\
0 & x_{i j}<0
\end{array} ; \quad x_{i j}^{(2)}= \begin{cases}0 & x_{i j} \geq 0 \\
-x_{i j} & x_{i j}<0\end{cases}\right.
$$

The following model is used to calculate efficiency of $\mathrm{DMU}_{o}$ :

$$
\begin{aligned}
& \operatorname{Max} E_{o}^{1}, \\
& \operatorname{Max} E_{o}^{2}, \\
& \text { s.t. } E_{j}^{(k)} \leq 1 ; \quad k=1,2 ; \quad j=1, \ldots, n .
\end{aligned}
$$

All variables are non-negative. There are different methods to solve the two-objective Model (9). In this study, fuzzy method [10] is applied. General algorithm of this method is presented in Section 5. By putting $k=2$ in the presented algorithm, Model (9) can be solved.

\section{5. $k$-stage NDEA-CCR model in the presence of semi-positive and semi-negative data}

Consider $n$ Decision Making Units (DMUs) under assessment, each having a network structure, as shown in Figure 2. Each DMU consists of $\mathrm{k}$ stages. Let $m_{k}$ and $r_{k}$ be the numbers of inputs and outputs of the $k$-th stage. Assuming that $r_{k}=\hat{r}_{k}+\bar{r}_{k}$ and $m_{k}=\hat{m}_{k} \cup \bar{m}_{k}$, where $\hat{r}_{k}$ represents the number of outputs, all DMUs are positive in the $k$-th stage and $\bar{r}_{k}$ indicates the number of outputs as some DMUs are positive while others are negative in the $k$-th stage. If the $k$ and $h$ stages are linked to each other, they are denoted by 


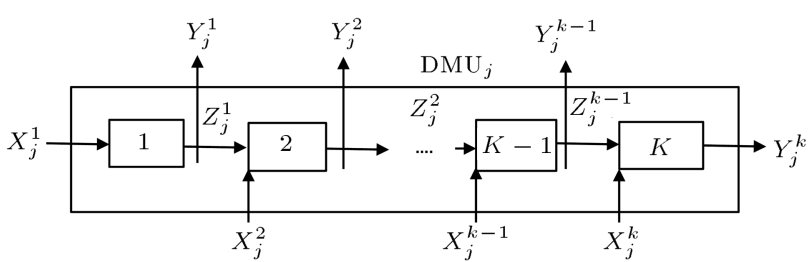

Figure 2. $k$-stage network model.

$(k, h)$. The intermediate product, $z_{j}^{(k, h)}$, which is an output of the $k$-th stage, plays input role for the $h$ th stage and its number is equal to $L_{k}$. Similarly, it can be written as $L_{k}=\hat{L}_{k}+\bar{L}_{k}$, where $\hat{L}_{k}$ shows the number of intermediate products, which is positive for the $k$-th stage in all DMUs, and $\bar{L}_{k}$ is the number of intermediate products, which is positive for the $k$-th stage in some DMUs and negative in others.

The multi-objective NDEA-CCR model in the presence of negative data is defined as follows:

$\operatorname{Max} E_{o}^{1}$

$$
\begin{aligned}
& \operatorname{Max} E_{o}^{k}=\frac{E_{o}^{K, Y}}{E_{o}^{K, X}}, \\
& k=1, \ldots, K \\
& E_{j}^{k} \leq 1 ; \quad k=1, \ldots, K ; \quad j=1, \ldots, n,
\end{aligned}
$$

where:

$$
\begin{aligned}
E_{j}^{k, Y}= & \sum_{r=1}^{\hat{r}_{k}} u_{r}^{k} y_{r j}^{k}+\sum_{r=1}^{\bar{r}_{k}} \bar{u}_{r}^{k} y_{r j}^{(1) k}-\sum_{r=1}^{\bar{r}_{k}} \hat{u}_{r}^{k} y_{r j}^{(2) k} \\
& +\sum_{\forall(k, h)} \sum_{p=1}^{t(k, h)} \mu_{h}^{k} z_{p j}^{(k, h)}+\sum_{\forall(k, h)} \sum_{p=1}^{\bar{t}(k, h)} \bar{\mu}_{h}^{k} z_{p j}^{(2)(k, h)} \\
& -\sum_{\forall(k, h)} \sum_{p=1}^{\bar{t}(k, h)} \hat{\mu}_{h}^{k} z_{p j}^{(2)(k, h)}, \\
E_{j}^{k, X}= & \sum_{i=1}^{\hat{m}_{k}} v_{i}^{k} x_{i j}^{k}+\sum_{i=1}^{\bar{m}_{k}} \bar{v}_{i}^{k} x_{i j}^{(1) k}-\sum_{i=1}^{\bar{m}_{k}} \hat{v}_{i}^{k} x_{i j}^{(2) k} \\
& +\sum_{\forall(g, k)} \sum_{q=1}^{t(g, k)} w_{g}^{t} z_{q j}^{(g, k)}+\sum_{\forall(g, k)} \sum_{q=1}^{\bar{t}(g, k)} \bar{w}_{g}^{k} z_{q j}^{(1)(g, k)} \\
& -\sum_{\forall(g, k)} \sum_{q=1}^{\bar{t}(g, k)} \hat{w}_{g}^{k} z_{q j}^{(2)(g, k)},
\end{aligned}
$$

where $y_{r j}^{k}=y_{r j}^{(1) k}-y_{r j}^{(2) k} ; \forall r \in\left\{1, \ldots, \bar{r}_{k}\right\} ; y_{r j}^{(1) k} \geq 0$; $y_{r j}^{(2) k} \geq 0$, where:

$$
y_{r j}^{(1) k}=\left\{\begin{array}{ll}
y_{r j}^{k} & y_{r j}^{k} \geq 0 \\
0 & y_{r j}^{k}<0
\end{array} ; \quad y_{r j}^{(2) k}= \begin{cases}0 & y_{r j}^{k} \geq 0 \\
-y_{r j}^{k} & y_{r j}^{k}<0\end{cases}\right.
$$

And $x_{i j}^{k}=x_{i j}^{(1) k}-x_{i j}^{(2) k} ; \forall i \in\left\{1, \ldots, \bar{m}_{k}\right\} ; x_{i j}^{(1) k} \geq 0$; $x_{i j}^{(2) k} \geq 0$, where:

$$
x_{i j}^{(1) k}=\left\{\begin{array}{ll}
x_{i j}^{k} & x_{i j}^{k} \geq 0 \\
0 & x_{i j}^{k}<0
\end{array} ; \quad x_{i j}^{(2) k}= \begin{cases}0 & x_{i j}^{k} \geq 0 \\
-x_{i j}^{k} & x_{i j}^{k}<0\end{cases}\right.
$$

And $z_{q j}^{(g, k)}=z_{q j}^{(1)(g, k)}-z_{q j}^{(2)(g, k)} ; \forall q \in\{1, \ldots, \bar{t}\}$; $z_{q j}^{(1)(g, k)} \geq 0 ; z_{q j}^{(2)(g, k)} \geq 0$, where:

$$
\begin{aligned}
& z_{q j}^{(1)(g, k)}=\left\{\begin{array}{ll}
z_{q j}^{(g, k)} & z_{q j}^{(g, k)} \geq 0 \\
0 & z_{q j}^{(g, k)}<0
\end{array} ;\right. \\
& z_{q j}^{(2)(g, k)}= \begin{cases}0 & z_{q j}^{(g, k)} \geq 0 \\
-z_{q j}^{(g, k)} & z_{q j}^{(g, k)}<0\end{cases}
\end{aligned}
$$

To solve the above model (Model (10)) through fuzzy method [10], the following operation is performed:

- Step 1. The ideal answer for each of the objective functions is obtained.

$$
\begin{aligned}
& E_{o}^{k *}=\max E_{o}^{k}, \\
& \text { s.t. } \quad E_{j}^{k} \leq 1, \quad j=1, \ldots, n .
\end{aligned}
$$

All weights are non-negative.

The optimal answer of Model (16) is calculated for $k=1, \ldots, K$ and its optimal value is named $E_{o}^{k *}$;

Step 2. The anti-ideal answer for each of the objective functions is obtained:

$$
\begin{aligned}
& E_{o}^{k-}=\min E_{o}^{k}, \\
& \text { s.t. } \quad E_{j}^{k} \geq 1, \quad j=1, \ldots, n .
\end{aligned}
$$

All weights are non-negative.

The optimal answer of Model (17) is calculated for $k=1, \ldots, K$ and its optimal value is named $E_{o}^{k-}$;

- Step 3. The membership function for each of the objective functions for ideal and anti-ideal answers is defined as follows:

$$
\mu\left(E_{o}^{k}\right)=\frac{E_{o}^{k}-E_{o}^{k-}}{E_{o}^{k *}-E_{o}^{k-}} .
$$

Note that since $E_{o}^{(k) *}$ is ideal efficiency of $\mathrm{DMU}_{o}$, $0 \leq E_{o}^{(k) *} \leq 1 . E_{o}^{(k)-}$, the distance to the border of inefficiency, is anti-ideal efficiency of $\mathrm{DMU}_{o}$. Therefore, $E_{o}^{(k)-} \geq 1$; since $E_{o}^{(k) *} \leq E_{o}^{(k)} \leq E_{o}^{(k)-}$, it can be easily proved for $E_{o}^{(k) *}-E_{o}^{(k)}-\neq 0$ and always 
$0 \leq \mu E_{o}^{(k)} \leq 1$. For the case of $E_{o}^{(k) *}-E_{o}^{(k)-}=0$, a unit is added to the denominator of $\mu E_{o}^{(k)}$ in order to overcome its problem in becoming zero denominator;

- Step 4. The following model is solved:

$$
\begin{aligned}
& \max \lambda=\min \left\{\mu\left(E_{o}^{k}\right)\right\}, \\
& \text { s.t. } \quad \lambda \leq \mu\left(E_{o}^{k}\right), \quad k=1, \ldots, K, \\
& \mu\left(E_{o}^{k}\right)=\frac{E_{o}^{k}-E_{o}^{k-}}{E_{o}^{k *}-E_{o}^{k-}}, \quad k=1, \ldots, K,
\end{aligned}
$$

for all original constraints;

- Step 5. $E_{o}=\sum_{k=1}^{k} w_{k} E_{o}^{k}$ is considered to be overall efficiency of $\mathrm{DMU}_{o}$ unit, where $w_{k}$ is weight of the $k$-th stage. $\sum_{k=1}^{k} w_{k}=1$. Note that since for each $k$, $0 \leq E_{o}^{(k)} \leq 1$, efficiency is always between $(0,1]$. if $E_{o}=1$, then we say that $\mathrm{DMU}_{o}$ is efficient; and for each $i \neq j$, if $E_{j} \geq E_{i}$, then the $i$-th unit is more efficient than the $j$-th unit.

\section{Case studies}

In this section, two numerical examples are investigated. In the first case, an example with two stages is evaluated. This data includes semi-positive and seminegative indices. In the second case, an example with $k$ stages is studied that makes a CCR-MOP model. The fuzzy method stated in Section 3 is used for finding the optimum answer.

\subsection{Case 1: Example of two-stage NDEA-CCR with semi-positive and semi-negative data}

In this section, units consisting of two stages are considered. According to Figure $3, X_{j}$ is the input of Stage 1 and its outputs are $Z_{j}$ and $\bar{Z}_{j}$, which are positive outputs and semi-positive and semi-negative outputs, respectively. These outputs serve as inputs to Stage 2. Also, $\bar{x}_{j}$ is external input to Stage 2. The outputs of Stage 2 are $Y_{j}$ and $\bar{Y}_{j}$, which are positive outputs and semi-positive and semi-negative outputs, respectively. This data is presented in Table 1 .

According to Table 1, Stage 1 includes three positive inputs and Stage 2 has two positive outputs, $y_{1 j}$, $y_{2 j}$, and two semi-positive and semi-negative outputs, $\bar{y}_{1 j}, \bar{y}_{2 j}$. Output of Stage 1 (input to Stage 2) includes two positive outputs, $z_{1 j}, z_{2 j}$, and two semi-positive and semi-negative outputs, $\bar{z}_{1 j}, \bar{z}_{2 j}$.

Model (8) is used to calculate efficiency of $\mathrm{DMU}_{0}$ $(o \in\{1, \ldots, 10\})$. In this model, $E_{j}^{1}$, and $E_{j}^{2}$ are calculated as follows:

$$
\begin{aligned}
& E_{j}^{1}=\frac{\mu z_{j}+\bar{\mu}_{1} \bar{z}_{1 j}^{(1)}-\hat{\mu}_{1} \bar{z}_{1 j}^{(2)}+\bar{\mu}_{2} \bar{z}_{2 j}^{(1)}-\hat{\mu}_{2} \bar{z}_{2 j}^{(2)}}{v_{1} x_{1 j}+v_{2} x_{2 j}}, \\
& E_{j}^{2}= \\
& \frac{u_{1} y_{1 j}+u_{2} y_{2 j}+\bar{u}_{1} \bar{y}_{1 j}^{(1)}-\hat{u}_{1} \bar{y}_{1 j}^{(2)}+\bar{u}_{2} \bar{y}_{2 j}^{(1)}-\hat{u}_{2} \bar{y}_{2 j}^{(2)}}{\mu z_{j}+\bar{\mu}_{1} \bar{z}_{1 j}^{(1)}-\hat{\mu}_{1} \bar{z}_{1 j}^{(2)}+\bar{\mu}_{2} \bar{z}_{2 j}^{(1)}-\hat{\mu}_{2} \bar{z}_{2 j}^{(2)}+\bar{v} \bar{x}_{j}},(20)
\end{aligned}
$$

where Model (8) for evaluation of $\mathrm{DMU}_{1}$ is as follows:

$\operatorname{Max} E_{o}^{1}$,

$\operatorname{Max} E_{o}^{2}$

\begin{tabular}{|c|c|c|c|c|c|c|c|c|c|c|}
\hline \multirow[b]{2}{*}{$\mathrm{DMU}_{j}$} & \multicolumn{3}{|c|}{ Stage 1} & \multicolumn{3}{|c|}{ Link } & \multicolumn{4}{|c|}{ Stage 2} \\
\hline & $x_{1 j}$ & $x_{2 j}$ & $\overline{\boldsymbol{x}}_{j}$ & $z_{j}$ & $\bar{z}_{1 j}$ & $\bar{z}_{2 j}$ & $y_{1 j}$ & $y_{2 j}$ & $\bar{y}_{1 j}$ & $\bar{y}_{2 j}$ \\
\hline 1 & 0.838 & 0.277 & 0.962 & 0.894 & 0.362 & -0.410 & 0.879 & 0.337 & 0.177 & -0.423 \\
\hline 2 & 1.233 & 0.132 & 0.443 & 0.678 & 0.188 & -0.932 & 0.538 & 0.180 & 0.915 & -0.240 \\
\hline 3 & 0.321 & 0.045 & 0.482 & 0.836 & -0.207 & 0.595 & 0.911 & 0.198 & -0.488 & 0.413 \\
\hline 4 & 1.483 & 0.111 & 0.467 & 0.869 & -0.516 & 0.518 & 0.570 & 0.491 & 0.437 & 0.547 \\
\hline 5 & 1.592 & 0.208 & 1.073 & 0.693 & -0.407 & 0.689 & 1.086 & 0.372 & -0.549 & -0.994 \\
\hline 6 & 0.790 & 0.139 & 0.545 & 0.966 & 0.269 & -0.918 & 0.722 & 0.253 & 0.401 & 0.398 \\
\hline 7 & 0.451 & 0.075 & 0.366 & 0.647 & 0.257 & 0.888 & 0.509 & 0.241 & -0.533 & 0.371 \\
\hline 8 & 0.408 & 0.074 & 0.229 & 0.756 & -0.103 & -0.474 & 0.619 & 0.097 & 0.522 & -0.825 \\
\hline 9 & 1.864 & 0.061 & 0.691 & 1.191 & 0.402 & 0.443 & 1.023 & 0.380 & -0.456 & 0.467 \\
\hline 10 & 1.222 & 0.149 & 0.327 & 0.792 & -0.187 & -0.674 & 0.769 & 0.178 & -0.309 & 0.702 \\
\hline
\end{tabular}

s.t. $\quad E_{j}^{(k)} \leq 1 ; \quad k=1,2 ; \quad j=1, \ldots, 10$.

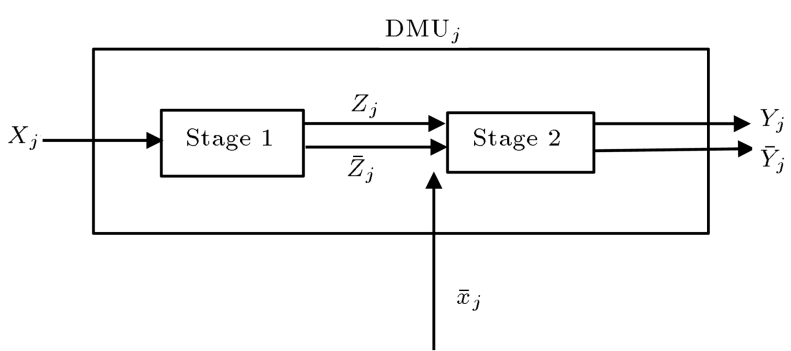

Figure 3. Two-stage network model with external input.

Table 1. The sample data of Case 1. 
Table 2. Ideal and anti-ideal efficiencies of units of Case 1.

\begin{tabular}{ccccccc}
\hline $\mathbf{D M U}_{j}$ & $\boldsymbol{E}_{j}^{(\mathbf{1}) *}$ & $\boldsymbol{E}_{j}^{(\mathbf{2}) *}$ & $\boldsymbol{E}_{j}^{(\mathbf{1})-}$ & $\boldsymbol{E}_{j}^{(\mathbf{2})-}$ & $\boldsymbol{E}_{j}^{(\mathbf{2}) *}-\boldsymbol{E}_{j}^{(\mathbf{1})-}$ & $\boldsymbol{E}_{j}^{(\mathbf{2}) *}-\boldsymbol{E}_{j}^{(\mathbf{2}) *}$ \\
\hline 1 & 0.75807 & 1 & 1.31914 & 1 & -0.56107 & $\varepsilon$ \\
2 & 0.43852 & 1 & 2.28041 & 1 & -1.84189 & $\varepsilon$ \\
3 & 1 & 0.99998 & 1 & 1 & $\varepsilon^{*}$ & $-2 \mathrm{E}-05$ \\
4 & 0.41578 & 0.9999 & 2.40515 & 1 & -1.98937 & $-1 \mathrm{E}-04$ \\
5 & 0.25053 & 1 & 3.99161 & 1 & -3.74108 & $\varepsilon$ \\
6 & 0.73791 & 1 & 1.35519 & 1 & -0.61728 & $\varepsilon$ \\
7 & 1 & 0.51801 & 1 & 1.08145 & & -0.56344 \\
8 & 0.71148 & 0.99972 & 1.40553 & 1 & -0.69405 & -0.00028 \\
9 & 1 & 0.99994 & 1 & 1 & $\varepsilon$ & $-6 \mathrm{E}-05$ \\
10 & 0.28545 & 1 & 3.50318 & 1 & -3.21773 & $\varepsilon$ \\
\hline
\end{tabular}

* $\varepsilon$ is small amount and positive.

In order to solve the two objective models above, the algorithm presented in Section 5 is used:

- Step 1. solve these two models:

$$
\begin{aligned}
& E_{1}^{(1) *}=\operatorname{Max} E_{1}^{(1)} \\
& \text { s.t. } \quad E_{j}^{(k)} \leq 1 ; \quad k=1,2 ; \quad j=1, \ldots, 10, \\
& E_{1}^{(2) *}=\operatorname{Max} E_{1}^{(2)}, \\
& \text { s.t. } \quad E_{j}^{(k)} \leq 1 ; \quad k=1,2 ; \quad j=1, \ldots, 10 .
\end{aligned}
$$

All variables are assumed non-negative in both models. Assume that $E_{1}^{(1) *}$ and $E_{1}^{(2) *}$ are ideal efficiencies of Stages 1 and 2 related to $\mathrm{DMU}_{o}$. The results of Models (22) and (23) are shown in Table 2 (note the first row of Table 2);

- Step 2. Solve the following models:

$$
\begin{aligned}
& E_{1}^{(1)-}=\operatorname{Min} E_{1}^{(1)} \\
& \text { s.t. } \quad E_{j}^{(1)} \geq 1 ; \quad k=1,2 ; \quad j=1, \ldots, 10, \\
& E_{1}^{(2)-}=\operatorname{Min} E_{1}^{(2)}, \\
& \text { s.t. } \quad E_{j}^{(1)} \geq 1 ; \quad k=1,2 ; \quad j=1, \ldots, 10 .
\end{aligned}
$$

All variables in both models are assumed nonnegative. Assume that $E_{1}^{(1)-}$ and $E_{1}^{(2)-}$ are antiideal efficiencies of Stages 1 and 2 related to $\mathrm{DMU}_{o}$, of which the results are given in the first row of Table 2.

- Step 3. The membership function for each of the Steps 1 and 2 using ideal and anti-ideal efficiency values is defined as follows:

$$
\begin{aligned}
& \mu\left(E_{1}^{(1)}\right)=\frac{E_{1}^{(1)}-E_{1}^{(1)-}}{E_{1}^{(1) *}-E_{1}^{(1)-}}, \\
& \mu\left(E_{1}^{(2)}\right)=\frac{E_{1}^{(2)}-E_{1}^{(2)-}}{E_{1}^{(2) *}-E_{1}^{(2)-}},
\end{aligned}
$$

where $0 \leq \mu\left(E_{1}^{(1)}\right) \leq 1,0 \leq \mu\left(E_{1}^{(2)}\right) \leq 1$.

- Step 4. Solve the following model:

$\operatorname{Max} \lambda$

$$
\begin{array}{ll}
\text { s.t. } & \lambda \leq \mu\left(E_{1}^{(1)}\right) \\
& \lambda \leq \mu\left(E_{1}^{(2)}\right),
\end{array}
$$

$\mu\left(E_{1}^{(k)}\right)=\frac{E_{1}^{(k)}-E_{1}^{(k)-}}{E_{1}^{(k) *}-E_{1}^{(k)-}} ; \quad k=1,2$,

$E_{1}^{(1)}=\frac{\mu z_{1}+\bar{\mu}_{1} \bar{z}_{11}^{(1)}-\hat{\mu}_{1} \bar{z}_{11}^{(2)}+\bar{\mu}_{2} \bar{z}_{21}^{(1)}-\hat{\mu}_{2} \bar{z}_{21}^{(2)}}{v_{1} x_{11}+v_{2} x_{21}}$,

$E_{1}^{(2)}=\frac{u_{1} y_{11}+u_{2} y_{21}+\bar{u}_{1} \bar{y}_{11}^{(1)}-\hat{u}_{1} \bar{y}_{11}^{(2)}+\bar{u}_{2} \bar{y}_{21}^{(1)}-\hat{u}_{2} \bar{y}_{21}^{(2)}}{\mu z_{1}+\bar{\mu}_{1} \bar{z}_{11}^{(1)}-\hat{\mu}_{1} \bar{z}_{11}^{(2)}+\bar{\mu}_{2} \bar{z}_{21}^{(1)}-\hat{\mu}_{2} \bar{z}_{21}^{(2)}+\bar{v}_{j}}$

$E_{j}^{(1)} \leq 1 ; \quad k=1,2 ; \quad j=1, \ldots, 10$.

All variables are non-negative;

- Step 5. Assume that $E_{1}^{(1)}$ and $E_{j}^{(2)}$ are results of optimum answer of Model (27). These results are given in Table 3 . In order to calculate efficiency of $\mathrm{DMU}_{1}$, correlation of $E_{1}=1 / 2 E_{1}^{(1)}+1 / 2 E_{1}^{(2)}$ is used. Not that results are given in Table 3 with consideration of $w_{1}=w_{2}=1 / 2$. 
Table 3. Overall efficiency of units of Case 1.

\begin{tabular}{|c|c|c|c|c|c|c|c|c|c|}
\hline $\mathrm{DMU}_{j}$ & $\nu_{1}$ & $\nu_{2}$ & $\nu$ & & $\boldsymbol{w}$ & $\overline{\boldsymbol{w}}_{1}$ & $\bar{w}_{2}$ & $\hat{w}_{1}$ & $\hat{w}_{2}$ \\
\hline 1 & $4.59 \mathrm{E} 3$ & 0 & $6.14 \mathrm{E} 4$ & & 0 & 8.05 E3 & 0 & 0 & 0 \\
\hline 2 & $9.71 \mathrm{E} 7$ & 0 & 6.97 E7 & & 0 & $1.70 \mathrm{E} 8$ & 0 & 0 & 0 \\
\hline 3 & $9.71 \mathrm{E} 7$ & 0 & $2.30 \times E 11$ & & $3.73 \mathrm{E} 7$ & $7.66 \mathrm{E} 7$ & 0 & 0 & 0 \\
\hline 4 & $9.71 \mathrm{E} 7$ & 0 & $9.90 \mathrm{E} 9$ & & $3.73 \mathrm{E} 7$ & 7.66 E7 & 0 & 0 & 0 \\
\hline 5 & $1.04 \mathrm{E} 7$ & 0 & 1.80 E10 & & $3.99 \mathrm{E} 7$ & $8.20 \mathrm{E} 7$ & 0 & 0 & 0 \\
\hline 6 & $2.92 \mathrm{E} 4$ & 0 & $6.03 \mathrm{E} 6$ & & $1.12 \mathrm{E} 4$ & $2.30 \mathrm{E} 4$ & 0 & 0 & 0 \\
\hline 7 & $2.07 \mathrm{E} 7$ & 0 & $1.46 \mathrm{E} 8$ & & 7.94 E6 & $1.63 \mathrm{E} 7$ & 0 & 0 & 0 \\
\hline 8 & $1.27 \mathrm{E} 5$ & 0 & $1.17 \mathrm{E} 2$ & & $4.89 \mathrm{E} 4$ & 1.00 E5 & 0 & 0 & 0 \\
\hline 9 & $5.08 \mathrm{E} 2$ & $8.40 \mathrm{E} 3$ & $1.08 \mathrm{E} 5$ & & $6.47 \mathrm{E} 2$ & $1.71 \mathrm{E} 3$ & 0 & 0 & 0 \\
\hline $\mathbf{D M U}_{j}$ & $u_{1}$ & $u_{2}$ & $\bar{u}_{1}$ & $\overline{\boldsymbol{u}}_{2}$ & $\hat{\boldsymbol{u}}_{1}$ & $\hat{\boldsymbol{u}}_{2}$ & $E_{j}^{(1)}$ & $E_{j}^{(2)}$ & $\begin{array}{l}\text { Overal efficiency } \\
\left(w_{1}=w_{2}=1 / 2\right)\end{array}$ \\
\hline 1 & $6.63 \mathrm{E} 4$ & $3.70 \mathrm{E} 3$ & $2.80 \mathrm{E} 4$ & 0 & $1.53 \mathrm{E} 4$ & $6.00 \mathrm{E} 3$ & 0.75807 & 1 & 0.879035 \\
\hline 2 & 0 & 0 & 7.07 E7 & 0 & $1.90 \mathrm{E} 4$ & $7.28 \mathrm{E} 6$ & 0.43852 & 1 & 0.71926 \\
\hline 3 & 0 & 0 & 1.30 E11 & 0 & 0 & $2.60 \mathrm{E} 10$ & 1 & 0.99998 & 0.99999 \\
\hline 4 & 0 & 0 & 1.10 E10 & 0 & 0 & 2.10 E10 & 0.41578 & 0.99998 & 0.70788 \\
\hline 5 & 0 & 0 & $1.70 \mathrm{E} 10$ & 0 & 0 & 0 & 0.25053 & 1 & 0.625265 \\
\hline 6 & $2.74 \mathrm{E} 6$ & $1.34 \mathrm{E} 6$ & $2.46 \mathrm{E} 6$ & $5.27 \mathrm{E} 3$ & 0 & $2.79 \mathrm{E} 6$ & 0.73791 & 1 & 0.868955 \\
\hline 7 & $6.33 \mathrm{E} 7$ & $1.25 \mathrm{E} 7$ & $4.16 \mathrm{E} 7$ & 5.27 E3 & 0 & $5.18 \mathrm{E} 7$ & 1 & 0.56077 & 0.780385 \\
\hline 8 & $3.13 \mathrm{E} 4$ & 0 & $3.39 \mathrm{E} 4$ & 3.66 E1 & 0 & 0 & 0.71148 & 0.99994 & 0.85571 \\
\hline 9 & $6.94 \mathrm{E} 4$ & 0 & $4.19 \mathrm{E} 4$ & $1.15 \mathrm{E} 4$ & 0 & 0 & 1 & 0.99999 & 0.999995 \\
\hline
\end{tabular}

According to Table 3, overall efficiency of none of the units is unit. Thus, $\mathrm{DMU}_{3}$ and $\mathrm{DMU}_{9}$ have higher efficiencies than other units.

\subsection{Case 2: Extended electric power companies}

This case is related to extended electric power companies with link 3 [19]. In Case 1 [19], inputs and outputs are not positive, but this case is an extension of case 1 with the exception that two semi-positive and semi-negative outputs are added to Stages 2 and 3 (to better understand, refer to Figure 4). Data of this case is given in Table 4 , where $X_{j}, \bar{X}_{j}$ and $\hat{X}_{j}$ are inputs of Stages 1, 2, and 3, respectively. $Y_{j}^{2}$ and $\bar{Y}_{j}^{2}$ are positive outputs and semi-positive and semi-negative outputs of Stage 2, respectively. $Y_{j}^{3}$ and $\bar{Y}_{j}^{3}$ are positive outputs and semi-positive and semi-negative outputs of Stage 3, respectively. Furthermore, $Z_{j}^{1}$ and $Z_{j}^{2}$ are links of Stage 1 to Stage 2 and Stage 2 to Stage 3, respectively, in which $Z_{j}^{1}$ and $Z_{j}^{2}$ have non-negative values:

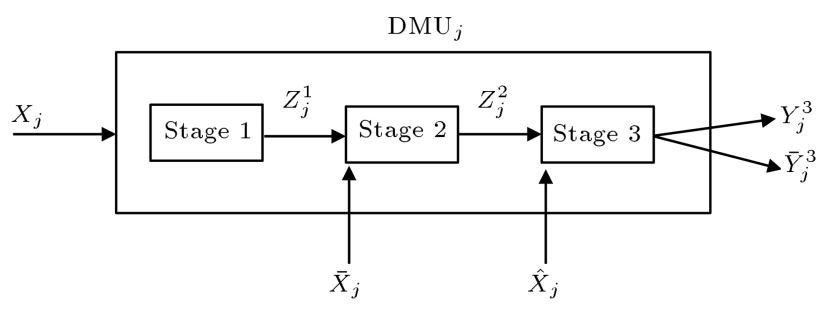

Figure 4. Three-stage network model.

$$
\begin{aligned}
& E_{j}^{(1)}=\frac{w z_{j}^{1}}{v x_{j}}, \\
& E_{j}^{(2)}=\frac{A z_{j}^{2}+u y_{j}^{2}+N \bar{y}_{j}^{2(1)}-D \bar{y}_{j}^{2(2)}}{w z_{j}^{1}+M \bar{x}_{j}}, \\
& E_{j}^{(3)}=\frac{P y_{j}^{3}+Q \bar{y}_{j}^{(3) 1}-S \bar{y}_{j}^{(3) 2}}{A z_{j}^{2}+B \hat{x}_{j}} .
\end{aligned}
$$

At first, Models (16) and (17) are used for data of Table 4. Ideal and ant-ideal answers are obtained for each unit and the results are presented in Table 5. Results of Model (19) for Case 2 are presented in Table 6.

The fuzzy method of Kao and his co-authors is used and the membership function value is calculated for each unit of assessment. Also, the overall efficiency of each $\mathrm{DMU}_{j}$ is calculated via $E_{j}=\sum_{k=1}^{3} w_{k} E_{j}^{k}$ with the assumption of $w_{1}=w_{2}=w_{3}=1 / 3$, and the results are presented in Table 7 . In this case, the weights of all the stages are assumed to be identical. As shown in Table $7, \mathrm{DMU}_{3}$ is efficient and the other DMUs are inefficient.

\section{Conclusion}

Measuring efficiency of units under assessment is one of the valuable goals of data envelopment analysis. Since all input and output indices cannot be positive, 
Table 4. The sample data of Case 2.

\begin{tabular}{|c|c|c|c|c|c|c|c|c|c|}
\hline \multirow[b]{2}{*}{$\mathbf{D M U}_{j}$} & \multirow{2}{*}{$\begin{array}{c}\text { Stage } 1 \\
\text { Input } 1 \\
\left(X_{j}\right)\end{array}$} & \multicolumn{3}{|c|}{ Stage 2} & \multicolumn{3}{|c|}{ Stage 3} & \multicolumn{2}{|c|}{ Link } \\
\hline & & $\begin{array}{c}\text { Input } 2 \\
\left(\bar{X}_{j}\right)\end{array}$ & $\begin{array}{c}\text { Output } 1 \\
\left(Y_{j}^{2}\right)\end{array}$ & $\begin{array}{c}\text { Output } 2 \\
\left(\bar{Y}_{j}^{2}\right)\end{array}$ & $\begin{array}{c}\text { Input } 3 \\
\left(\bar{X}_{j}\right)\end{array}$ & $\begin{array}{c}\text { Output } 3 \\
\left(Y_{j}^{\mathbf{3}}\right)\end{array}$ & $\begin{array}{c}\text { Output } 4 \\
\left(\bar{Y}_{j}^{3}\right)\end{array}$ & $\begin{array}{c}\text { Link } 1 \\
\left(Z_{j}^{1}\right)\end{array}$ & $\begin{array}{c}\text { Link } 2 \\
\left(Z_{j}^{2}\right)\end{array}$ \\
\hline 1 & 0.838 & 0.277 & 0.879 & 0.903 & 0.962 & 0.337 & 0.936 & 0.894 & 0.362 \\
\hline 2 & 1.233 & 0.132 & 0.533 & 0.097 & 0.443 & 0.180 & -0.188 & 0.578 & 0.188 \\
\hline 3 & 0.321 & 0.045 & 0.911 & 1.355 & 0.482 & 0.198 & 0.906 & 0.836 & 0.207 \\
\hline 4 & 1.483 & 0.111 & 0.570 & 1.621 & 0.467 & 0.491 & 0.812 & 0.869 & 0.516 \\
\hline 5 & 1.592 & 0.208 & 1.086 & -1.932 & 1.073 & 0.372 & -0.092 & 0.693 & 0.407 \\
\hline 6 & 0.790 & 0.139 & 0.722 & -0.737 & 0.545 & 0.253 & 0.064 & 0.966 & 0.269 \\
\hline 7 & 0.451 & 0.075 & 0.509 & 0.284 & 0.366 & 0.241 & 0.650 & 0.647 & 0.257 \\
\hline 8 & 0.408 & 0.074 & 0.619 & 1.094 & 0.229 & 0.097 & -1.150 & 0.756 & 0.103 \\
\hline 9 & 1.864 & 0.061 & 1.023 & 0.531 & 0.691 & 0.380 & 0.380 & 1.191 & 0.402 \\
\hline 10 & 1.222 & 0.149 & 0.769 & -0.026 & 0.337 & 0.178 & -0.005 & 0.792 & 0.187 \\
\hline
\end{tabular}

Table 5. Ideal and anti-ideal efficiencies of units of Case 2.

\begin{tabular}{ccccccc}
\hline $\mathbf{D M U}_{j}$ & $\boldsymbol{E}_{j}^{(\mathbf{1}) *}$ & $\boldsymbol{E}_{j}^{(\mathbf{2}) *}$ & $\boldsymbol{E}_{j}^{(\mathbf{3}) *}$ & $\boldsymbol{E}_{j}^{(\mathbf{1})-}$ & $\boldsymbol{E}_{j}^{(\mathbf{2})-}$ & $\boldsymbol{E}_{j}^{(\mathbf{3})-}$ \\
\hline 1 & 0.40963 & 1 & 0.97325 & 2.45077 & 1 & 1.01044 \\
2 & 0.21114 & 0.7878 & 1 & 1.26321 & 1.2844 & 1.17199 \\
3 & 1 & 1 & 1 & 5.98289 & 12.53931 & 1.18488 \\
4 & 0.225 & 1 & 1 & 1.34613 & 5.17403 & 3.03264 \\
5 & 0.16714 & 1 & 0.95533 & 1 & 1.64535 & 1 \\
6 & 0.46951 & 0.65585 & 0.98436 & 2.80905 & 1.63687 & 1.339 \\
7 & 0.55084 & 0.91166 & 0.98362 & 3.29562 & 2.13869 & 1.8993 \\
8 & 0.71148 & 0.85394 & 0.98416 & 4.25668 & 2.63603 & 1.22178 \\
9 & 0.24534 & 1 & 0.99045 & 1.46783 & 5.2849 & 1.58622 \\
10 & 0.24886 & 0.89274 & 0.99703 & 1.48889 & 1.62641 & 1.52352 \\
\hline
\end{tabular}

Table 6. Results of Model (19) for Case 2.

\begin{tabular}{cccccccccccccc}
\hline DMU $_{j}$ & $\boldsymbol{W}$ & $\boldsymbol{V}$ & $\boldsymbol{A}$ & $\boldsymbol{U}$ & $\boldsymbol{N}$ & $\boldsymbol{D}$ & $\boldsymbol{M}$ & $\boldsymbol{P}$ & $\boldsymbol{Q}$ & $\boldsymbol{S}$ & $\boldsymbol{B}$ & $\boldsymbol{W}$ \\
\hline 1 & 0.4582 & 1.19332 & 0.88437 & 0.31301 & 0 & 0 & 2.1313 & 1.01104 & 0.35708 & 0 & 0.70671 & 0.4582 \\
2 & 0.31141 & 0.81103 & 1.79514 & 0.01349 & 0 & 0 & 5.97623 & 3.30896 & 0 & 0 & 1.49552 & 0.31141 \\
3 & 1.19617 & 3.11526 & 0 & 0.3017 & 0.53517 & 0 & 0 & 0 & 1.10375 & 0 & 2.07469 & 1.19617 \\
4 & 0.25892 & 0.67431 & 1.36079 & 0 & 0.18373 & 0 & 6.98201 & 2.03666 & 0 & 0 & 0.63776 & 0.25892 \\
5 & 0.24119 & 0.62814 & 1.22564 & 0.03792 & 0 & 0 & 4.00412 & 1.73229 & 0 & 0 & 0.46707 & 0.24119 \\
6 & 0.48604 & 1.26582 & 1.34837 & 0.26357 & 0 & 0 & 3.81644 & 2.5292 & 0 & 0 & 1.16934 & 0.48604 \\
7 & 0.85138 & 2.21729 & 2.17825 & 0.49233 & 0 & 0 & 5.98878 & 2.43018 & 0.60643 & 0 & 1.20271 & 0.85138 \\
8 & 0.94111 & 2.45098 & 0 & 0 & 0.71012 & 0 & 3.89897 & 4.15336 & 0 & 0 & 4.36681 & 0.94111 \\
9 & 0.20599 & 0.53648 & 2.47192 & 0 & 0.01185 & 0 & $1.24 \mathrm{E}+01$ & 2.60643 & 0 & 0.00216 & 0.0091 & 0.20599 \\
10 & 0.31422 & 0.81833 & 0.77865 & 0.36044 & 0 & 0 & 5.04122 & 3.22966 & 0 & 0 & 2.53529 & 0.31422 \\
\hline
\end{tabular}

extended models are proposed by which the efficiency of units can be calculated. Also, some methods have been presented for measuring two- and multi-stage network DEA structures, which are applied for positive data. The difference between this article and other articles that focus on calculating units with two and multi stages is that a model is proposed in this paper, which is able to calculate the efficiency of network DEA in the presence of semi-positive and semi-negative indices. Two case studies for the presented work were presented. 
Table 7. Overall efficiency of Case 2 results of Model 19.

\begin{tabular}{ccccc}
\hline $\mathbf{D M U}_{j}$ & $\boldsymbol{E}_{j}^{(\mathbf{1})}$ & $\boldsymbol{E}_{j}^{(\mathbf{2})}$ & $\boldsymbol{E}_{j}^{(\mathbf{3})}$ & $\boldsymbol{E}_{\boldsymbol{j}}$ \\
\hline 1 & 0.40963 & 0.59528 & 0.67495 & 0.559953 \\
2 & 0.21114 & 0.34474 & 0.59561 & 0.38383 \\
3 & 1 & 1 & 1 & 1 \\
4 & 0.225 & 1 & 1 & 0.741667 \\
5 & 0.16714 & 0.54002 & 0.64441 & 0.450523 \\
6 & 0.46951 & 0.55301 & 0.63989 & 0.554137 \\
7 & 0.55084 & 0.8104 & 0.97985 & 0.780363 \\
8 & 0.71148 & 0.77688 & 0.40288 & 0.630413 \\
9 & 0.24534 & 1 & 0.99045 & 0.745263 \\
10 & 0.24886 & 0.42278 & 0.57488 & 0.415507 \\
\hline
\end{tabular}

In the first example, units with two stages having semipositive and semi-negative indices were considered; in the second example, units with 3 stages were selected. This ensured that some outputs of Stage 2 and 3 were semi-negative and semi-positive. Thereafter, the proposed method was applied to calculate the overall efficiency of units under assessment. To calculate the overall efficiency of units with more than two stages, solving MOP model was necessary. Fuzzy programming method as a solution procedure was proposed. The question that arises is whether it can be offered as a method to calculate the overall efficiency of multistage NDEA, so that solving MOP problem would not be needed.

Izadikhah and Farzipoor Saen [16] presented a model in order to calculate efficiency of two-stage network in the presence of negative data. Their twostage network structure consisted of input, output, and intermediate indices. The strength of the proposed method was calculating efficiency of $k$ th-stage network in the presence of negative data. Thus, those networks had external input to the $k$-th stage in addition to consumed input of Stage 1.

In future research, Multi-Objective Programming (MOP) can be applied in the presence of interval data, which is not crisp. Such problems can be solved using fuzzy techniques. Furthermore, this research can be expanded for solving MOP with consideration of interval negative data.

\section{Nomenclature}

$e_{j} \quad$ Efficiency of the $j$-th stage

$e_{j}^{(1)}, e_{j}^{(2)} \quad$ Efficiency of the first and second stages

$E_{j}^{k, X} \quad$ Efficiency of the $k$-th stage in input related to $\mathrm{DMU}_{j}$

$E_{k} \quad$ Efficiency of the $k$-th stage

$E_{o}^{K}$
$E_{o}^{k *}$
$E_{o}^{k-}$
$E_{o}^{K, X}$
$E_{o}^{K, Y}$
$I$
$\bar{I}$

$L_{k}$

$\bar{L}_{k}$

$\hat{L}_{k}$

$\bar{r}_{k}$

$\hat{r}_{k}$

$R$

$\bar{R}$

$T$

$\bar{T}$

$u_{o}$

$u_{r}, \bar{u}_{r}, \hat{u}_{r}$

$u_{r}^{k}$

$\bar{u}_{r}^{k}$

$v_{i}, \bar{v}_{i}, \hat{v}_{i}$

$\bar{v}_{i}^{k}, \hat{v}_{i}^{k}, v_{i}^{k}$

$\bar{w}_{g}^{k}, \hat{w}_{g}^{k}$

$w_{g}^{t}$

$w_{k}$

$x_{i j}$
Efficiency of the $k$-th stage related to $\mathrm{DMU}_{0}$

The ideal efficiency of the $k$-th stage related to $\mathrm{DMU}_{o}$

The anti-ideal efficiency of the $k$-th stage related to $\mathrm{DMU}_{0}$

Efficiency of the $k$-th stage in input related to $\mathrm{DMU}_{o}$

Efficiency of the $k$-th stage in output related to $\mathrm{DMU}_{0}$

Input series, which is positive in all DMUs

Input series, which is positive in some DMUs and negative in other DMUs

The number of intermediate products

The number of intermediate products, which is positive for the $k$-th stage in some DMUs and negative in others

The number of intermediate products, which is positive for the $k$-th stage in all DMUs

The number of outputs when some DMUs are positive and others are negative in the $k$-th stage

The number of outputs when all DMUs are positive in the $k$-th stage

Output series, which is positive in all DMUs

Output series, which is positive in some DMUs and negative in others $z_{t j}$ series, which is positive in all DMUs $z_{t j}$ series, which is positive in some units and negative in others

Constant return to scale

Weights of outputs, which are non-negative

Output weight of the $k$-th stage

Output weight of the $k$-th stage for semi-positive and semi-negative

Weights of inputs which are nonnegative

Input weights of the $k$-th stage

Intermediate weight of the $k$-th stage for semi-positive and semi-negative inputs

Intermediate weight of the $t$-th stage, which is positive

Weight of the $k$-th stage

Input to the $j$-th stage 
$x_{i j}^{(1)}, x_{i j}^{(2)}$

$x_{i j}^{k}$

$x_{i j}^{(1) k}, x_{i j}^{(2) k}$

$x_{i k}$

$x_{i o}$

$x_{i o}^{(1)}, x_{i o}^{(2)}$

$X_{j}$

$\bar{X}_{j}$

$y_{r j}$

$y_{r j}^{(1)}, y_{r j}^{(2)}$

$y_{r j}^{(1) k}, y_{r j}^{(1) k}$

$y_{r j}^{k}$

$y_{r k}$

$y_{\text {ro }}$

$y_{\text {ro }}^{(1)}, y_{\text {ro }}^{(2)}$

$Y_{j}$

$\bar{Y}_{j}$

$Z_{j}$

$\bar{Z}_{j}$

$z_{p j}^{(k, h)}$

$z_{p j}^{(1)(k, h)}, z_{p j}^{(2)(k, h)}$

$z_{q j}^{(g, k)}$

$z_{q j}^{(1)(g, k)}, z_{q j}^{(2)(g, k)}$ Intermediate stage related to $\mathrm{DMU}_{j}$ from $k$ and $g$ loops, which is from semi-positive and semi-negative data sets

$z_{t j}$

$z_{t} j^{(1)}, z_{t} j^{(2)}$

Input to the $j$-th stage when $\bar{I}$ belongs to the negative and positive sets

Input to the $k$-th stage related to $\mathrm{DMU}_{j}$

Input to the $j$-th stage for semi-positive and semi-negative

Input to the $k$-th stage

Input to the stage related to $\mathrm{DMU}_{o}$

Input to the stage related to $\mathrm{DMU}_{o}$ when $\bar{I}$ belongs to negative and positive sets

Input to Stage 1, which is positive

Input to Stage 1, which is semi-positive and semi-negative

Output of the $j$-th stage

Output of the $j$-th stage when $\bar{R}$ belongs to negative and positive sets

Output of the $k$-th stage related to $\mathrm{DMU}_{j}$ for semi-positive and semi-negative

Output of the $k$-th stage related to $\mathrm{DMU}_{j}$

Output of the $k$-th stage

Output of the stage related to $\mathrm{DMU}_{o}$

Output of the stage related to $\mathrm{DMU}_{o}$ when $\mathrm{DMU}_{o}$ belongs to negative and positive sets

Outputs of Stage 2, which is positive

Outputs of Stage 2, which is semi-positive and semi-negative Output of Stage 1, which is positive

Output of Stage 1, which is semipositive and semi-negative

Intermediate product related to the $k$-th and $h$-th stages for $\mathrm{DMU}_{j}$ $h$-th stages related to $\mathrm{DMU}_{j}$ Intermediate stage related to $\mathrm{DMU}_{j}$ from $k$ and $g$ loops, which is positive Intermediate variable of the $j$-th stage Intermediate variable of the $j$-th stage when $\bar{T}$ belongs to negative and positive sets
$\mu\left(E_{o}^{k}\right)$

$\bar{\mu}_{h}^{k}$

$\mu_{h}^{k}$

$\mu_{t}, \bar{\mu}_{t}, \hat{\mu}_{t}$

$\varepsilon$

The membership function for each of the objective functions for ideal and anti-ideal answers related to $\mathrm{DMU}_{o}$ Intermediate weight of the $k$-th stage Intermediate weight of the $k$-th stage Weights of intermediate stage, which are non-negative

Positive and small variable

\section{References}

1. Tone, K. and Tsutsui, M. "Network DEA: A slacksbased measure approach", Eur. J. Oper. Res., 197(1), pp. 243-252 (2009).

2. Sexton, T.R. and Lewis, H.F. "Two-stage DEA: An application to major league baseball", J. Prod. Anal. 19(2), pp. 227-249 (2003).

3. Despotis, D.K. and Koronakos, G. "Efficiency assessment in two-stage processes: A novel network DEA Approach", Pro. Computer Science, 31, pp. 299-307 (2014).

4. Carayannis, E.G., Goletsis, Y. and Grigoroudis, E. "Multi-level multi-stage efficiency measurement: the case of innovation systems", Oper. Res., 15(2) pp. 253274 (2015).

5. Jarosz, P., Kusiak, J., MaBecki, S.B., Oprocha, P., Sztangret, A. and Wilkus, M.A. "Methodology for optimization in multistage industrial processes: A Pilot Study", Math. Prob. Eng., 2015, pp. 1-10 (2015).

6. Gang, D., Li, C., Yin-Zhen, L. and Jie-Yan, S.A. "Tanweer, optimization on production-inventory problem with multistage and varying demand", J. Appl. Math., 2012, pp. 1-17 (2012).

7. Charnes, A., Cooper, W.W. and Rhodes, E. "Measuring the efficiency of decision making units", Eur. J. Oper. Res., 2(6), pp. 429-444 (1978).

8. Banke, R., Charnes, A. and Cooper, W.W. "Some models for estimating technical and scale inefficiencies in data envelopment analysis", Manag. Sci., 30(9), pp. 1078-1092 (1984).

9. Cheng, H., Zhang, Y., Cai, J. and Huang, W. "A multiobjective programming method for ranking all units based on compensatory DEA model", Math. Prob. Eng., 2014, pp. 1-14. (2014).

10. Kao, H.Y., Chan, C.Y. and Wu, D.J. "A multiobjective programming method for solving network DEA", Appl. Soft Comput., 24(2014), pp. 406-413 (2014).

11. Kazemi Matin, R. and Azizi, R. "A unified networkDEA model for performance measurement of production systems", Measurement., 60, pp. 186-193 (2015).

12. Wang, W.K., Lu, W.M. and Liu, P.Y. "A fuzzy multiobjective two-stage DEA model for evaluating the 
performance of US bank holding companies", Expert. Syst. Appl., 41(9), pp. 4290-4297 (2014).

13. Dimitris, K. and Despotis, G.K. "Efficiency assessment in two-stage processes: A novel network DEA approach", Procedia. Comput. Sci., 31, pp. 299-307 (2014).

14. Halkos, G.E., Tzeremes, N.G. and Kourtzidis, S.A. "A unified classification of two-stage DEA models", Sur. Oper. Res. Manag. Sci., 19(1), pp. 1-16 (2014).

15. Lee, E.S. and Li, R.J. "Fuzzy multiple objective programming and compromise programming with Pareto optimum", Fuzzy Set. Syst., 53(3), pp. 275-288 (1993).

16. Izadikhah, M. and Farzipoor Saen, R. "Evaluating sustainability of supply chains by two-stage range directional measure in the presence of negative data", Trans. Res. Part D., 49, pp. 110-126 (2016).

17. Olfata, L., Amiri, M., Soufi, J.B. and Pishdar, M. "A dynamic network efficiency measurement of airports performance considering sustainable development concept: A fuzzy dynamic network-DEA approach", $J$. Air. Trans. Manag., 57, pp. 272-290 (2016).

18. Chen, Y. and Zhu, J. "Measuring information technology's indirect impact on firm performance", Inform. Tech. Manag. J., 5(1-2) pp. 9-22 (2004).

19. Tone, K. and Tsutsui, M. "Network DEA: a slacksbased measure approach", Eur. J.Oper. Res 197, pp. 243-252 (2009).

\section{Biographies}

Kianoosh Kianfar is $\mathrm{PhD}$ candidate in Industrial Engineering with specialty in Operations Research and Systems Engineering at Science and Research Branch, Islamic Azad University, Tehran, Iran. His research interests are industrial engineering, data envelopment analysis, operations research, and decision theory. He has published a manuscript in the International Journal of Data Envelopment Analysis based on his research activities.

Mahnaz Ahadzadeh Namin received his $\mathrm{PhD}$ degree in Applied Mathematics from Science and Research Branch, Islamic Azad University, Tehran, Iran, in 2009. She is currently Assistant Professor at Shahre-Qods Branch, Islamic Azad University. Her research interests are data envelopment analysis, efficiency analysis, applied mathematics, productivity analysis, and applied econometrics.

Akbar Alam Tabriz received his $\mathrm{PhD}$ degree in Management in Turkey in 1989. He is currently Associate Professor at Shahid Beheshti University. His research interests are performance evaluation in production industries, implementation of Total Quality Management (TQM), and efficiency and productivity measurement in industry. He has performed research and published some papers in the mentioned fields.

Esmaeil Najafi received his $\mathrm{PhD}$ degree in Industrial Engineering with specialty in Management and System Efficiency from Science and Research Branch, Islamic Azad University, Tehran, Iran, in 2009. He is currently Associate Professor at Science and Research Branch, Islamic Azad University. His research interests are industrial engineering, applied and computational mathematics, operations management, and optimization.

Farhad Hosseinzadeh Lotfi received his $\mathrm{PhD}$ degree in Applied Mathematics from Science and Research Branch, Islamic Azad University, Tehran, Iran, in 2000. He is currently Professor at Science and Research Branch, Islamic Azad University. His research interests are mathematics, operations research, data envelopment analysis, and efficiency. 